\title{
Negative Tension of Scroll Wave Filaments and Turbulence in Three-Dimensional Excitable Media and Application in Cardiac Dynamics
}

\author{
Sergio Alonso • Markus Bär • Alexander V. Panfilov
}

Received: 15 December 2011 / Accepted: 28 June 2012 / Published online: 25 July 2012

(C) Society for Mathematical Biology 2012

\begin{abstract}
Scroll waves are vortices that occur in three-dimensional excitable media. Scroll waves have been observed in a variety of systems including cardiac tissue, where they are associated with cardiac arrhythmias. The disorganization of scroll waves into chaotic behavior is thought to be the mechanism of ventricular fibrillation, whose lethality is widely known. One possible mechanism for this process of scroll wave instability is negative filament tension. It was discovered in 1987 in a simple two variables model of an excitable medium. Since that time, negative filament tension of scroll waves and the resulting complex, often turbulent dynamics was studied in many generic models of excitable media as well as in physiologically realistic models of cardiac tissue. In this article, we review the work in this area from the first simulations in FitzHugh-Nagumo type models to recent studies involving detailed ionic models of cardiac tissue. We discuss the relation of negative filament tension and tissue excitability and the effects of discreteness in the tissue on the filament tension. Finally, we consider the application of the negative tension mechanism to computational cardiology, where it may be regarded as a fundamental mechanism that explains differences in the onset of arrhythmias in thin and thick tissue.
\end{abstract}

Keywords Reaction-diffusion systems · Cardiac tissue

\section{Introduction}

The main feature of an extended excitable medium is the conduction of propagating waves. Velocity and shape of such waves are determined by the properties of the

\footnotetext{
S. Alonso $(\varangle) \cdot$ M. Bär

Physikalisch-Technische Bundesanstalt, Abbestrasse 2-12, 10587 Berlin, Germany

e-mail: sergio.alonso@ptb.de
}

\section{A.V. Panfilov}

Department of Physics and Astronomy, Gent University, Krijgslaan 281, S9 9000 Gent, Belgium 
medium (Meron 1992). At certain conditions, such waves can form vortices which take on the shape of spiral waves rotating around a small core area. Such spiral waves were first predicted in simple models of cardiac tissue (Wiener and Rosenblueth 1946). Experimentally, they were at first discovered (Winfree 1972) in the chemical Belousov-Zhabotinsky reaction (Zaikin and Zhabotinsky 1970). Nowadays, spiral waves are found in excitable media of various nature in physics, chemistry, and biology (Keener and Sneyd 1998).

One of the most relevant examples for spiral waves in nature are rotating electrical excitations in cardiac tissue. Contraction of the heart is controlled by electrical waves of excitation initiated by the natural pacemaker. Such waves propagate through the myocardium and induce the contraction of the cardiac fibers. Abnormal excitation of the heart results in cardiac arrhythmias. Ventricular tachycardia (VT) is a common cardiac arrhythmia that is characterized by a high rate of cardiac excitation and, therefore, a high rate of contraction of the heart. Experimental and clinical studies show that in many cases tachycardia is driven by a spiral wave of excitation (Gray et al. 1995; Weiss et al. 2000). Ventricular tachycardia can deteriorate into ventricular fibrillation (VF). VF is characterized by a turbulent pattern of excitation that disrupts the coordinated contraction of the heart and often results in sudden cardiac death. The transition of a single spiral state into turbulent pattern is referred to as spiral breakup and was studied in models of cardiac tissue for more than 20 years (Panfilov and Holden 1990; Bär and Eiswirth 1993; Karma 1993; Panfilov and Hogeweg 1993; Panfilov and Pertsov 2001). Possible scenarios of spiral breakup in models of cardiac tissue are surveyed in Panfilov and Pertsov (2001) and Fenton et al. (2002). The most discussed mechanisms include dynamic instabilities, e.g., those induced by steep restitution curves (Panfilov and Holden 1990; Karma 1993), heterogeneity (Moe et al. 1964), and more recently mechano-electric feedback (Panfilov et al. 2007). However, the exact mechanism of onset of chaotic activity patterns in the heart in real conditions remains unknown. Currently, researchers believe that the mechanism of VF may be different in distinct conditions, and thus studying of various mechanisms of onset of turbulence in excitable medium is important (Fenton et al. 2002). It is widely accepted that one of the factors important for the onset of VF is the three-dimensional (3D) structure of the heart. It was even suggested that VF can occur only if the thickness of the ventricular wall of the heart exceeds some critical value (Winfree 1994); it could imply that at least in some cases VF is generated by essentially 3D instability mechanisms.

The extension of a two-dimensional spiral wave into a third dimension is a scroll wave. Scroll waves have been observed in many excitable systems, e.g., in the Belousov-Zhabotinsky chemical reaction (Winfree 1973), in the slug phase of the life cycle of slime molds (Siegert and Weijer 1992), and in the ventricles of the heart during cardiac arrhythmias (Pertsov and Jalife 2002). Numerous modeling studies of scroll waves have been performed using analytical and numerical methods. A scroll wave is usually characterized by its filament. The filament can be regarded as the line connecting the rotation centers of the spirals, which can be detected in the twodimensional sections of the scroll wave. This filament can be straight or curved; it can even be closed into a ring, giving rise to a scroll ring.

Filaments are dynamical structures which can move in space organizing the 3D dynamics of vortices in the medium. As a result of the dynamics, the length of a 
filament may increase or decrease. Decrease of the filament length is connected with a positive filament tension, while increase of the length is the result of a negative filament tension. It turns out that negative filament tension implies an instability of a straight scroll wave and that the growth of the filament length amounts to an increase of the overall vorticity in the medium. The aim of this paper is to describe the main results on negative filament tension, their possible relation to turbulence in excitable media, and project it to cardiac tissue.

The manuscript is organized as follows. After a basic description of excitable media given in the next section (Sect. 2), we will describe in Sect. 3 the phenomenon of negative filament tension in simple models of excitable media aimed at describing electrical propagation in cardiac tissue qualitatively. In Sect. 4, we present several theoretical approaches to analyze negative filament tension. In Sect. 5, we focus on different control methods of negative filament tension instability in simple models. Finally, in Sect. 6, we extend the discussion to detailed electrophysiological models of cardiac tissue and consider the relevance of negative filament tension to cardiac arrhythmias.

\section{Modeling Approaches}

Generic excitable media are usually described by a system of two coupled reactiondiffusion equations:

$$
\begin{aligned}
\frac{\partial V}{\partial t} & =k I\left(V, w, p_{i}\right)+\nabla \cdot\left(D_{V} \nabla V\right), \\
\frac{\partial w}{\partial t} & =R\left(V, w, p_{i}\right)+\nabla \cdot\left(D_{w} \nabla w\right) .
\end{aligned}
$$

In chemical excitable medium, all reacting species diffuse. In cardiac tissue, the diffusion operator is present only in the first equation $\left(D_{w}=0\right)$ and $V$ describes the transmembrane potential. Below, two main classes of models will be described and discussed, namely simple models of excitable media consisting of a small number of reaction-diffusion equations which are computationally very efficient, describe cardiac excitation in a qualitative way, as well as detailed ionic models, which contain all relevant details of the electrophysiology of a specific type of cardiac cell and are typically based on direct measurements of ionic currents in cardiac tissue.

The numerical simulations are typically performed in a cubic domain with noflux boundary conditions with a size of several wave lengths. In such domains, the influence of the boundaries on the dynamics of the filaments is usually negligible. To study the effect of the thickness on the filament dynamics, a few simulations were performed in a slab with variable thickness. We also did a few simulations in domains of more complex shapes, representing a dog heart geometry.

\subsection{Simple Models of Excitable Media for Cardiac Dynamics}

Simple models for cardiac tissue typically consist of a reduced number of equations, between two and four, aimed to reproduce general qualitative properties of 
cardiac excitation, such as generation and propagation of a pulse, refractory properties, simple dispersion relations, etc. Some of that models are obtained as reduction of ionic models (Fenton and Karma 1998a), and some have the functions $I\left(V, w, p_{i}\right)$ and $R\left(V, w, p_{i}\right)$ as simple as possible (Izhikevich and FitzHugh 2006; Barkley et al. 1990). Such systems of typically two coupled equations are not derived from physiological data and some properties of the waves often differ from waves of transmembrane potential in physiologically realistic models. There are also adaptable models where $I\left(V, w, p_{i}\right)$ and $R\left(V, w, p_{i}\right)$ are complex expressions on $V$ and $w$ with several tuning parameters. Such models can quantitatively reproduce the overall characteristics of cardiac tissue important for propagation, such as restitution of action potential duration (Aliev and Panfilov 1996; Fenton and Karma 1998a) and restitution of conduction velocity (Fenton and Karma 1998a).

The main advantage of these models is their simplicity and ability to explain basic properties of cardiac tissue. These models are convenient to implement because the nonlinearities are not strong and allow the implementation of effective numerical solvers. Furthermore, the results obtained with such models are not restricted to an individual excitable system but are generic and may be applicable to the dynamics of excitable waves in other media. Analytical results are also possible for some of these models.

There are several generic models of excitable media which were used to study dynamics of scroll waves: The Puschino model (Panfilov and Rudenko 1987) and its modification (Biktashev et al. 1994) and the Barkley model (Dowle et al. 1997) became more popular. In this paper, we will illustrate many results using the Barkley model for excitable medium. Equivalent results can be obtained with other simple models. This model is formed by a couple of dimensionless reaction-diffusion equations:

$$
\begin{aligned}
\frac{\partial V}{\partial t} & =\frac{1}{\epsilon} V(1-V)\left(V-\frac{w+p_{2}}{p_{1}}\right)+\nabla \cdot\left(D_{V} \nabla V\right), \\
\frac{\partial w}{\partial t} & =V-w ;
\end{aligned}
$$

where $\epsilon$ is the ratio of the temporal scales between $V$ and $w$. The parameters $p_{1}$ and $p_{2}$ specify the activator kinetics, with $p_{2}$ effectively controlling the excitation threshold of the system.

Equations (2) correspond to the continuous limit of the reaction-diffusion system. The structure of the tissue is, however, discrete and the continuous reaction-diffusion model may have to be substituted by a discrete model. A large amount of cells connected by gap junctions forms the tissue. The discreteness can be accounted by the description of the tissue in terms of a grid of cells coupled by resistors. For the same model considered above, we obtain (Alonso et al. 2011):

$$
\begin{aligned}
\dot{V}_{i} & =\frac{1}{\epsilon} V_{i}\left(1-V_{i}\right)\left(V_{i}-\frac{w+p_{2}}{p_{1}}\right)+L\left(V_{i}, V_{j}\right), \\
\dot{w}_{i} & =V_{i}-w_{i},
\end{aligned}
$$


where $V_{i}$ is the action membrane potential of the cell $i$ and the function

$$
L\left(V_{i}, V_{j}\right)=\sum_{j}^{6} \eta_{i j}\left(V_{j}-V_{i}\right),
$$

is the sum of the coupling with the first neighbors in 3D. The parameter $\eta_{i j}$ is the strength of the coupling between cells $i$ and $j$ and it decreases with the resistivity between cells. For constant and large values of $\eta_{i j}$ the spatial operator $L\left(V_{i}, V_{j}\right)$ corresponds to the discrete version of the Laplacian operator $\left(L\left(V_{i}, V_{j}\right)=\nabla \cdot\left(D_{V} \nabla V\right)\right)$, and Eqs. (2) are recovered.

\subsection{Detailed Electrophysiological Models}

Electro-physiologically detailed models employ a description based on direct experimental observations derived from voltage clamp and patch clamp studies, and generally include many Hodgkin-Huxley type equations (Hodgkin and Huxley 1952) to describe individual ionic currents forming the total current $I\left(V, w, p_{i}\right)=\sum_{j} I_{j}$ that cross the cell membrane (Noble and Rudy 2001). Modern ionic models also describe the changes of concentrations of all major ions inside cardiac cells, and generally consist of anywhere between 10 and 60 equations (Luo and Rudy 1991; ten Tusscher et al. 2004). The quantitative descriptions of ionic currents in cardiac tissue are continually being revised, and there is not yet general agreement on the best model for all circumstances (ten Tusscher et al. 2006). The nonlinear terms are typically stiff and the integration is slower than in the previous cases.

Most results on scroll wave dynamics in ionic models here will be illustrated with the Luo-Rudy model phase 1 (LR1) (Luo and Rudy 1991). It describes the biophysical mechanism of generation of action potential in cardiac cells by a relatively small number of state variables and was widely used to study wave propagation in $2 \mathrm{D}$ and 3D cardiac tissue. Following the cable equation (Keener and Sneyd 1998), the propagation of a transmembrane potential can be described by the equation:

$$
\frac{\partial V}{\partial t}=-\frac{I_{\text {ion }}\left(V, w, p_{i}\right)}{C_{m}}+\nabla \cdot\left(D_{V} \nabla V\right) .
$$

The membrane capacitance is $C_{m}=1 \mathrm{~F} / \mathrm{cm}^{2}$ and the diffusion coefficient $D_{V}=$ $0.001 \mathrm{~cm}^{2} / \mathrm{ms}$ is derived from the resistivity between cells, surface to volume ratio of a cardiac cell and the membrane capacitance (Keener and Sneyd 1998). The representation of the total current is: $I_{\text {ion }}=I_{\mathrm{Na}}+I_{\mathrm{si}}+I_{K}+I_{K 1}+I_{\mathrm{Kp}}+I_{b}$, where $I_{\mathrm{Na}}=G_{\mathrm{Na}} m^{3} h j\left(V-E_{\mathrm{Na}}\right)$ is the fast $\mathrm{Na}^{+}$current, $I_{\mathrm{si}}=G_{\mathrm{si}} d f\left(V-E_{\mathrm{si}}\right)$ is the slow inward $\mathrm{Ca}^{2+}$ current, $I_{K}=G_{K} x x_{1}\left(V-E_{K}\right)$ is the slow outward $\mathrm{K}^{+}$current, $I_{K_{1}}=$ $G_{K_{1}} K_{1 \infty}\left(V-E_{K_{1}}\right)$ is the time-independent $\mathrm{K}^{+}$current, $I_{\mathrm{Kp}}=G_{\mathrm{Kp}} K_{p}\left(V-E_{\mathrm{Kp}}\right)$ is the plateau $\mathrm{K}^{+}$current, and $I_{b}=G_{b}\left(V-E_{b}\right)$ is the background current. The values $E_{\mathrm{Na}}, E_{\mathrm{si}}, E_{K}, E_{K_{1}}, E_{\mathrm{Kp}}$, and $E_{b}$ are the reversal potentials and $m, h, j, d, f$, and $x$ are gating variables, which dynamics can be modeled by

$$
\frac{d w_{i}}{d t}=\left(w_{i \infty}-w_{i}\right) / \tau_{w_{i}}
$$

where $w_{i}$ represents any of the gating variables, $w_{i \infty}$ is the steady state value, and $\tau_{w_{i}}$ the relaxation time constant for the corresponding variable. In most simulations 
presented here, the original values of the parameters given in Luo and Rudy (1991) were used, except for $G_{\mathrm{Na}}$ and $G_{\mathrm{si}}$, which were varied in simulations (in Luo and Rudy $1991 G_{\mathrm{Na}}=23 \mathrm{mS} / \mathrm{cm}^{2}$ and $G_{\mathrm{si}}=0.09 \mathrm{mS} / \mathrm{cm}^{2}$ ). Note that $G_{\mathrm{Na}}$ controls the speed of the waves (Alonso and Panfilov 2007) and in a general sense the excitability of the tissue, and $G_{\mathrm{si}}$ is responsible for the front-tail interaction between the pulses.

\section{Negative Filament Tension in Simple Qualitative Models}

Negative filament tension was found in the 1980s in computational studies of generic models of excitable media. At that time, only simplified models were feasible for 3D computational studies.

\subsection{Instability of the Filament and Turbulence}

A scroll wave in a thin slab, with its straight filament orthogonal to the boundaries, is a quasi 2D solution, as each section orthogonal to the filament is the same and all spatial derivatives in the third direction parallel to the filament will be zero. The first essential $3 \mathrm{D}$ effects occur if one considers either curved of twisted filaments. An ideal object to study the effect of curvature is a scroll ring that contains a circular filament, with constant curvature. The filament dynamics of scroll rings were studied in Panfilov and Pertsov (1984), Panfilov and Rudenko (1987) and Panfilov and Holden (1993). It was shown that the rotation of a scroll ring is nonstationary; see Fig. 1. The position of its filament is not stable and it drifts in space. The drift velocities are proportional to the filament curvature:

$$
\begin{aligned}
& V_{n}=-D_{n} k \\
& V_{b}=D_{b} k ;
\end{aligned}
$$

where $V_{n}$ and $V_{b}$ are drift velocities in normal (horizontal in Fig. 1) and binormal (vertical) directions, $D_{n}$ and $D_{b}$ are proportionality coefficients, and $k>0$ is the filament curvature. For scroll rings, $k$ is equal to $R^{-1}$, where $R$ is the radius of the ring and (7) takes the simple form:

$$
\begin{aligned}
& \dot{R}=-\frac{D_{n}}{R}, \\
& \dot{Z}=\frac{D_{b}}{R} .
\end{aligned}
$$

Based on the sign of the normal component of the velocity, two regimes of the scroll ring are distinguished. The first regime with positive $D_{n}$ corresponds to a contraction of the scroll ring (Panfilov and Pertsov 1984); see Fig. 1(b). When the ring filament reaches a critical radius, collapse of the scroll ring occurs. The second regime with $D_{n}<0$ is the scroll ring extension (Panfilov and Rudenko 1987) shown in Fig. 1(c). Under such conditions, the radius of the scroll ring increases proportionally to its curvature. Expansion and collapse of scroll rings are typically accompanied by a vertical drift; see Figs. 1(b)-(c).

Filament contraction obviously decreases the filament length, and thus was associated with positive tension, while extension of the filament corresponds to the 
a)

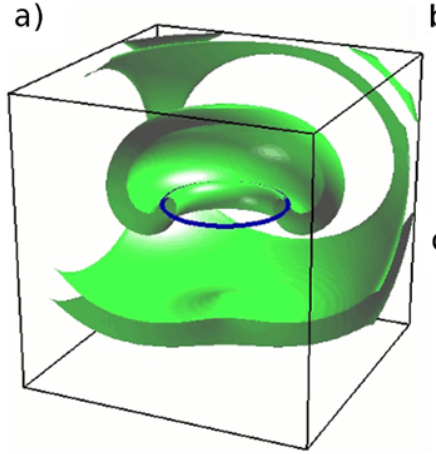

b)

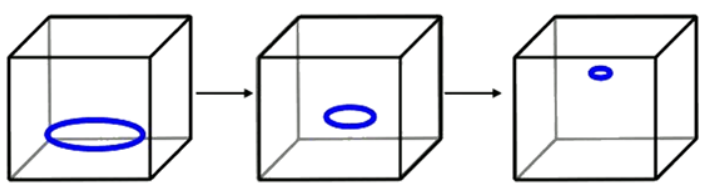

c)

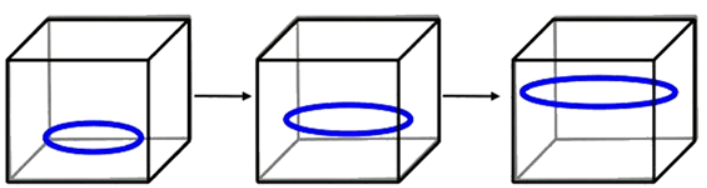

Fig. 1 (a) Scroll ring where waves and filament are visible. Half of the wave has been removed to permit the view of the filament. Sketch of the dynamics of a filament ring under positive (b) and negative (c) tension

a)

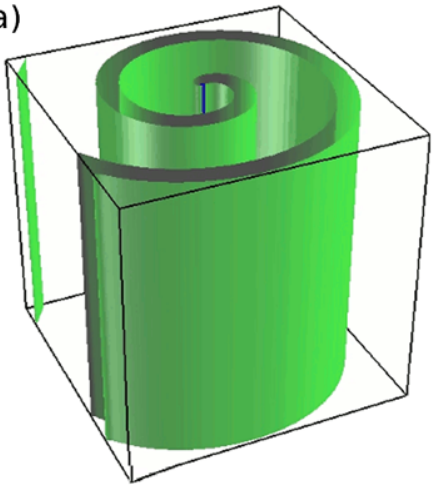

b)

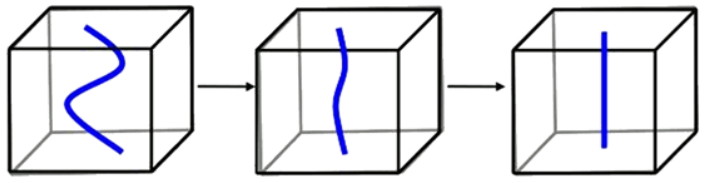

c)

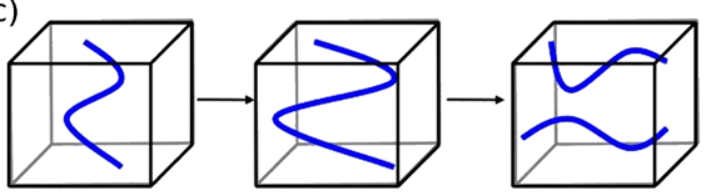

Fig. 2 (a) Scroll wave where waves and filament are visible. Sketch of the dynamics of the filament under positive (b) and negative (c) tension after a perturbation

case of negative tension. Tension, therefore, affects significantly the stability of the scroll wave filament. If the filament has positive tension and contracts, it will either collapse (e.g., for the case of a scroll ring) or it will attain a stable shape with the minimal possible length (e.g., for a straight scroll). For example, in a rectangular slab of isotropic cardiac tissue, it would evolve into a straight line orthogonal to the opposite tissue boundaries; see Fig. 2. Also, an initially perturbed straight filament will recover the stable straight configuration; see Fig. 2(b). However, if the filament has negative tension it tends to increase its length; see Fig. 2(c). It means it will form loops and if the filament touches a boundary, it will result in the filament breaking into two pieces, leading to filament multiplication (Biktashev et al. 1994; Fenton et al. 2002; Alonso et al. 2003; Gray and Jalife 1998). This regime has received different names from different authors, e.g., autowave turbulence (Biktashev 1998), scroll wave turbulence (Zaritski et al. 2004), or Winfree turbulence (Alonso et al. 2003). In simple models, positive tension occurs for a medium with high excitability, i.e., low excitation threshold and/or slow inhibitor dynamics, while 
Fig. 3 Parameter space of the Barkley model. Solid lines separate regions of positive filament tension, negative filament tension, bistability, and the subexcitable region (spiral waves are not observed). Dashed line encloses the meandering region. Modified version from Alonso et al. (2003)

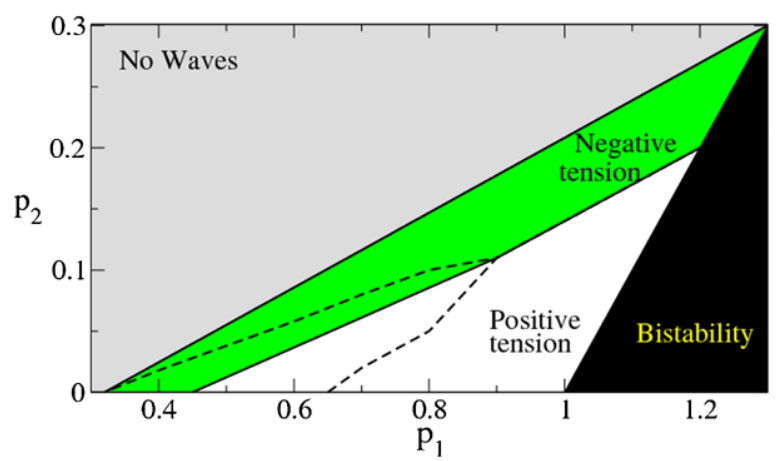

a)
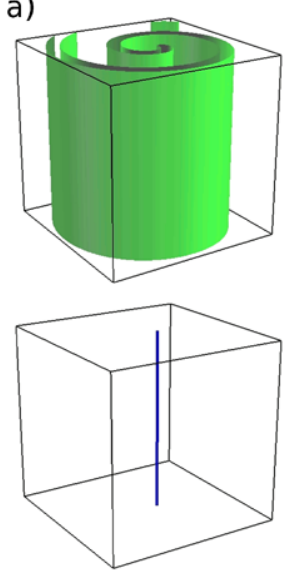

b)
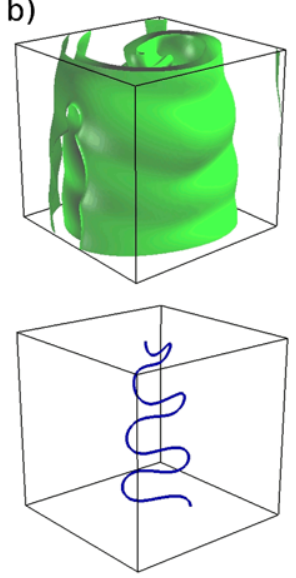

c)
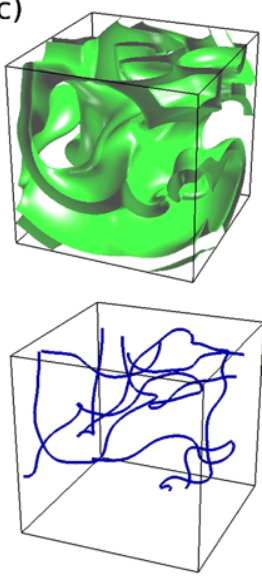

d)
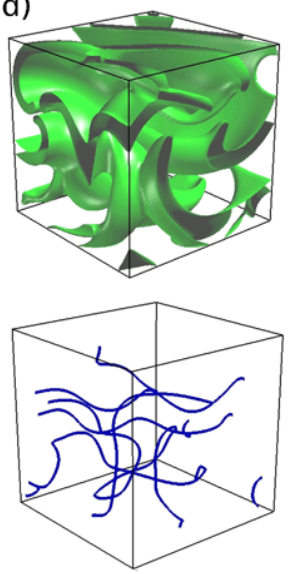

Fig. 4 Evolution of a scroll wave showing negative filament tension in the Barkley model. Waves and filaments are shown in different panels for the same time step

negative tension occurs for a medium with low excitability (Brazhnik et al. 1987; Fenton et al. 2002; Alonso et al. 2003).

The most detailed study of the onset of negative filament tension was performed in the Barkley model (2). For many parameter values, Eqs. (2) produce stable scroll waves with positive tension and without any scroll breakup; see Fig. 3. The negative filament tension was observed inside a broad region, characterized by low excitability. The regions in parameter space corresponding to negative and to positive filament tension are comparable in size. The negative tension region is located in the region of lower excitability near the boundary of the subexcitable region (No Waves in Fig. 3), exactly at the boundary the spiral rotation period increases to infinity. Negative filament tension results typically in scroll turbulence.

An example of turbulent dynamics is shown in Fig. 4. First, a scroll wave rotates around its straight filament (Fig. 4(a)). As time goes on, transverse deformations in the filament develop (Fig. 4(b)). The length of the filament rapidly increases because it loops out and forms an irregular expanding tangle. The tangle initially still consists of a single connected filament until the moment it touches a boundary plane. After 

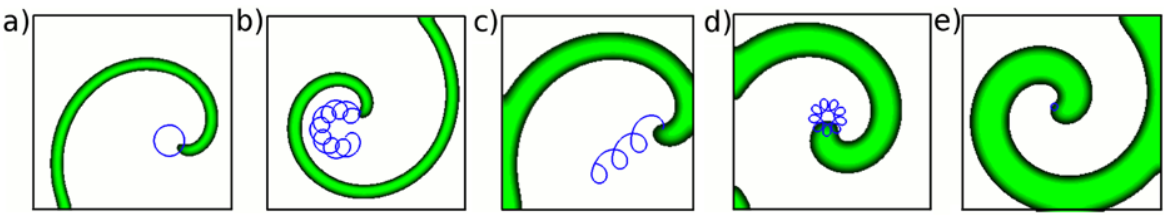

Fig. 5 Types of spiral waves observed in simulations with the Barkley model. (a) Rigidly rotating spiral wave with large core, (b) meandering spiral wave with inward loops, (c) drift meandering spiral, (d) meandering spiral wave with outward loops, (e) rigidly rotating spiral wave with small core

that, the filament gets fragmented into many pieces (Fig. 4(c)) that fill the medium and the resulting waves exhibit a turbulent appearance (Fig. 4(d)).

Upon onset of the instability, the total length of the filament increases exponentially with time. The total length saturates when the outflow of filament through the boundaries is compensated by the increase of filament length generated by the instability (Alonso et al. 2004a). The turbulent dynamics frequently presents an intermittent behavior between complex turbulence and a relatively organized state (Zaritski et al. 2004). This occurs due to the stabilizing effect of filament interaction, filaments tend to bunch in group of three or more filaments; see, e.g., filaments in Fig. 4(d). These filament bundles push the remaining individual pieces of filament outside the medium, and thereby counteracts the effects of negative filament tension. This effect is associated with parameters for which one finds formation of multiarmed spiral waves in two spatial dimensions, which typically occurs at low excitability conditions (Zaritski and Pertsov 2005).

Figure 3 displays also the region of spiral wave meandering. Numerical simulations of simple models point toward an intriguing relation between filament tension and meandering that we will elaborate on in the following passage. Spiral wave meandering is illustrated in Fig. 5. In general, spiral waves may rotate rigidly; see Figs. 5(a), (e), or may perform a cycloidal motion composed of two frequencies, known as meandering; see Figs. 5(b)-(d). The loops forming the meandering motion can be oriented toward the center (inward meandering); see Fig. 5(b), or outside (outward meandering), see Fig. 5(d). In the transition between both regions, the meandering spiral wave performs a linear drift; see Fig. 5(c).

The onset of the meandering is associated with a Hopf bifurcation of rigidly rotating spirals in excitable media (Karma 1990). One would not necessary expect any relation between meandering of $2 \mathrm{D}$ spiral waves and the tension of filaments in $3 \mathrm{D}$ excitable media at first glance. However, numerical simulations show that the meandering for parameter choices that display inward in $2 \mathrm{D}$, always negative filament tension in $3 \mathrm{D}$ is found. This relation has been observed in simple models of excitable media (Fenton et al. 2002; Alonso et al. 2004a, 2006a; Henry 2004). While outward meandering of spiral waves in 2D media corresponds in three dimensions to collapse of scroll rings, inward meandering in contrast is correlated with expansion of scroll rings in 3D.

This result seems independent of the origin of the meandering. Spiral waves under constant conditions meander because of their internal dynamics. However, meandering may as well be induced by an external periodic forcing (Mantel and Barkley 1996). Forcing with a frequency smaller (larger) than the frequency of spiral wave 
a)
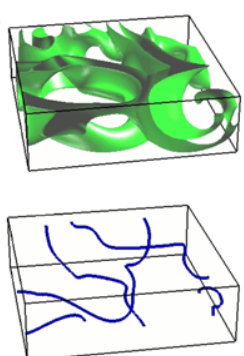

b)
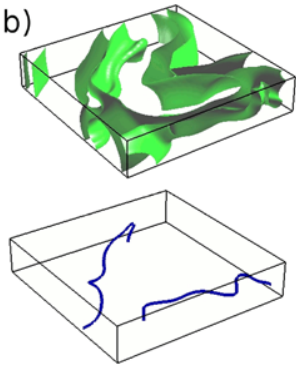

c)
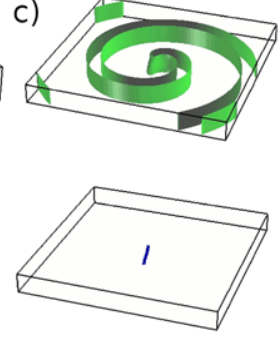

d)
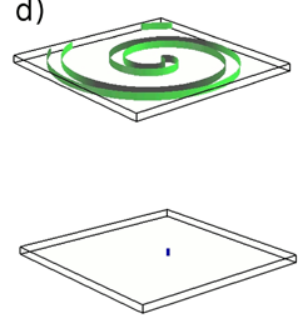

Fig. 6 Snapshots of waves and filaments at the end of simulations with the Barkley model under negative filament tension for four different thicknesses (measured in model units): 30 (a), 20 (b), 10 (c), and 4 (d)

rotation produces inward (outward) spiral meandering in 2D. In the corresponding $3 \mathrm{D}$ case, forcing with frequencies smaller than the period of the rigidly rotating spiral increases the filament tension and may induce positive filament tension for intense enough forcing, while forcing with frequencies larger than the spiral period decreases the filament tension and may induce negative filament tension (Alonso et al. 2003, 2006b). For more details, see Sect. 5.

\subsection{Size and Shape of Cardiac Tissue}

The instability due to negative filament tension and the resulting turbulence is a purely three-dimensional effect. For the same parameter values, spiral waves are stable in two dimensions. It follows that there is a minimal thickness for the three-dimensional wall below which the negative filament tension should disappear. In Fig. 6, we show the results of some numerical simulations with the same parameter values as in Fig. 4 but for different vertical thicknesses. For thick three-dimensional tissue the negative tension instability develops, see Fig. 6(a), with similar properties as in Fig. 4. However, when the tissue becomes very thin, the instability does not develop and the scroll wave rotates in a similar fashion as a spiral wave rotates in two-dimensional media; see Fig. 6(d). For intermediate thickness, the filament is unstable, however, the length of the filament saturates. The filament bends and undergoes a complex meandering motion without breaking up; see Fig. 6(c).

There is a critical thickness of the media to obtain the growth of the filament length resulting from negative filament tension. Slightly above this threshold, the filament is unstable and the filament length grows; however, it interacts with the boundaries and large regions of the filament remain parallel to the tissue; see Fig. 6(b).

Excitability of the medium can be decreased not only by changing the parameters of the model, but also as a result of a replacement of the partial different equation by a discrete model. In the context of cardiac tissue, this can be motivated by assuming that the cells are coupled to the others by gap junctions (Kléber and Rudy 2004). Suppose, we consider a grid of discrete cells coupled by resistors (see Eqs. (3)), and increase the resistivity between neighboring cells, i.e., we decrease the parameter $\eta_{i j}$ in Eq. (4). It is known that if the resistivity is large enough, waves cannot propagate along the tissue (Shaw and Rudy 1997). Such reduced coupling has similar effects as too low excitability conditions, wherein waves do not propagate in the medium. In 
a)
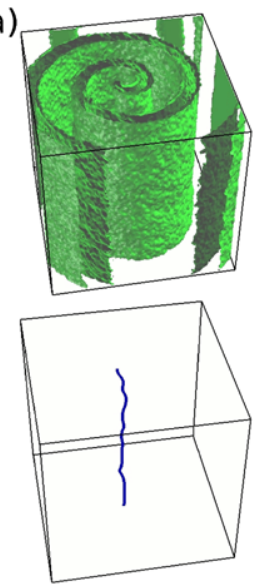

b)
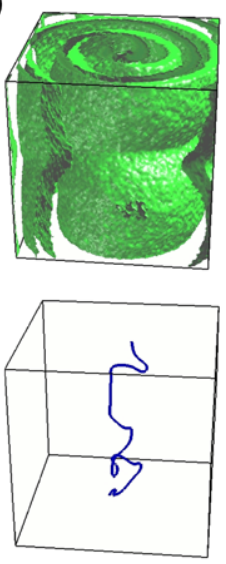

c)
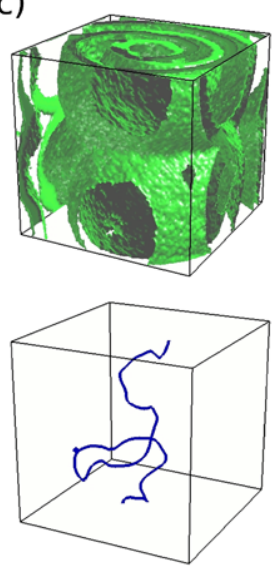

d)
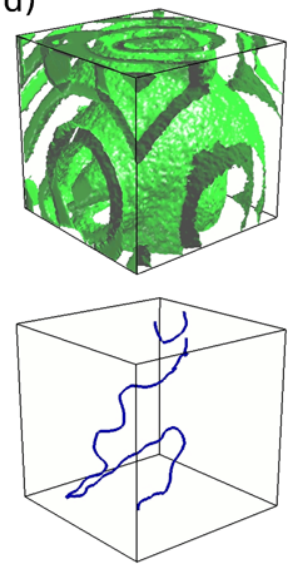

Fig. 7 Evolution of waves and filament of a scroll wave using the Barkley model under negative filament tension induced by heterogeneous discrete coupling among the cells of the tissue

many forms of cardiac disease (e.g., ischemia, infarction, etc.), intercellular conductance is substantially reduced, making the discrete nature of wave propagation more pronounced. The effects of discreteness upon the tension of filaments of scroll waves was studied in detail in Alonso et al. (2011).

Coupling among cells was reduced in two ways (Alonso et al. 2011): First, a fraction of the connections among the cells $\eta_{i j}$ are randomly decreased close to zero, producing that the effective coupling among the cells is reduced (Alonso et al. 2009), and second, the coupling among cells is homogeneous decreased $\eta_{i j}=\eta_{0}$. Numerical simulations with Eqs. (3) were performed under parameter values inside the positive tension region. Under such conditions, scroll rings contract and scroll waves are stable. A fraction of coupling among elements was reduced up to $10 \%$ of the original value. For low fraction of reduced connections, a scroll wave rotates stable, apart from some irregularities produced by the heterogeneous connection matrix. The period of rotation of the waves increases with the increase of the fraction of reduced connections (Panfilov 2002). An increase of the period of rotation is a signature of excitability decrease. It may produce a decrease on the filament tension (Alonso et al. 2011). Figure 7 shows that a scroll wave which is stable for large coupling may become unstable if a fraction of the couplings between cells is decreased. The filament length of the scroll wave grows (see Fig. 7(b)), and due to this negative filament tension instability we observe irregular wave behavior; see Figs. 7(c)-(d). The dynamics is similar to the results obtained with negative filament tension in an homogeneous system; see Fig. 4.

If the coupling among cells is homogeneously decreased the resulting dynamics is similar as in the previous case. For large values of the connectivity, the continuous limit is satisfied, therefore, scroll waves are completely stable. If the intercellular connections are homogeneously reduced, the rotation period of the wave increases when the characteristic size of the front is of the order of the distance among elements (Clayton et al. 2011). Then the filament attains negative tension and becomes 
a)

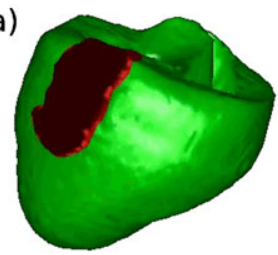

b)

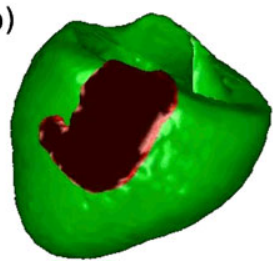

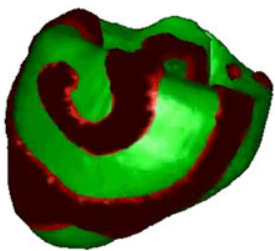
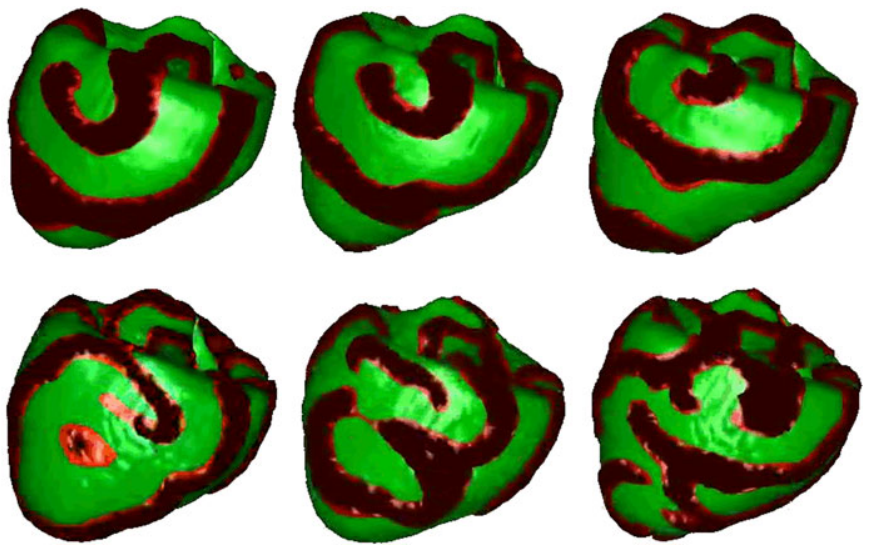

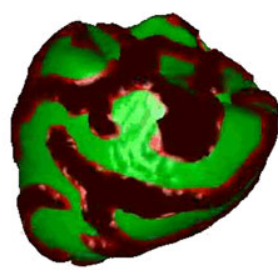

Fig. 8 Numerical simulations of wave propagation in a detailed dog heart geometry. Numerical simulations have been performed with the Aliev-Panfilov model (Aliev and Panfilov 1996) under positive (a) and negative (b) filament tension conditions. Both simulations employ the same initial condition

unstable, resulting in the onset of scroll wave turbulence (Alonso et al. 2011). For low connectivity, the propagation of waves is not more possible.

In both cases, a decreased coupling results in a decrease of filament tension. Such reduction may finally result in a qualitative change in the scroll wave dynamics: Initially stable scroll waves may become unstable and result in spatiotemporal chaotic wave patterns.

Simple generic models of excitable media were also employed to study wave propagation in anatomical models of the heart (Panfilov and Keener 1995a; Panfilov 1999). Recently, we studied the process of negative filament tension turbulence in the Aliev-Panfilov model (Aliev and Panfilov 1996) in an anatomical model of a dog heart (Nielsen et al. 1991; Panfilov 1999). Numerical results of scroll wave evolution for positive and negative filament tensions are shown in Fig. 8. For positive filament tension, a scroll wave is stable although it drifts slowly, see Fig. 8(a). A scroll wave with negative filament tension is unstable and produces wave turbulence; see Fig. 8(b). Interestingly enough, the new wave source appears as a point source below the cardiac surface; see Fig. 8(b) and the dynamics progresses into wave turbulence. Such scenario is similar to the transition of VT into VF observed in the heart (Zaitsev 2008).

\section{Theory on Filament Dynamics}

In the wake of the first simulations showing scroll ring expansion (Panfilov and Rudenko 1987), negative filament tension and the expansion of scroll rings were described in terms of a kinematic theory for the motion of curves (Brazhnik et al. 1987). It was also predicted that negative filament tension may result in turbulent behavior (Brazhnik et al. 1987). Later, the dynamics of curved and twisted filaments was studied using a perturbation theory (Keener 1988). A further important advance was the numerical calculation of the linear stability spectrum of scroll waves and the characterization of the eigenmodes corresponding to the negative filament tension (Henry 
and Hakim 2000). Next, we summarize the main concepts of such theoretical analysis of negative filament tension.

\subsection{Kinematic Theory}

In a three-dimensional system, a scroll wave is approximated by an oriented traveling surface with an open edge corresponding to the filament (Mikhailov 1995). Each element of the surface moves with a velocity proportional to the mean curvature $H$ of the surface: $V=V_{0}-2 D H$. The motion of the free edge of the surface depends on the mean curvature near the edge $\left(H_{0}\right)$ and on the geodetic curvature $\kappa$, and it grows $(G>0)$ or contracts $(G<0)$ with a velocity $G=G_{0}-2 \gamma H_{0}-\gamma^{\prime} \kappa$. The five kinematic parameters describing the evolution of a scroll wave $\left(V_{0}, G_{0}, \mathrm{D}, \gamma\right.$ and $\gamma^{\prime}$ ) can be determined from the particular reaction-diffusion model (Mikhailov et al. 1994).

The motion of the waves follows the dynamics of the free edge corresponding to the filament and, therefore, a quasisteady approximation can be applied (Mikhailov et al. 1994). For the particular case of scroll rings, the geodetic and mean curvatures can be expressed in terms of the angle of rotation around the filament $\alpha$ and the radius $R$ of the scroll ring: $\kappa=-R^{-1} \cos (\alpha)$ and $H_{0}=\left(k-R^{-1} \sin (\alpha)\right) / 2$. As a result a set of four ordinary differential equations for the position of the free edge ( $\rho$ and $z$ ), its local curvature $(c)$ and the angle of rotation $(\alpha)$ can be derived for the case of scroll rings with large radius (Brazhnik et al. 1987; Mikhailov 1995):

$$
\begin{aligned}
& \dot{c}=\frac{\omega_{0}}{D}\left(G_{0}-\gamma c+\frac{1}{R}\left(\gamma \sin \alpha+\left(\gamma^{\prime}-D\right) \cos \alpha\right)\right), \\
& \dot{\alpha}=\omega_{0}+c\left(G_{0}-\gamma c\right)+\frac{1}{R}\left(\gamma \sin \alpha+\left(\gamma^{\prime}-D\right) \cos \alpha\right) c, \\
& \dot{\rho}=-V_{0} \sin \alpha-\frac{1}{R}\left(D+\left(\gamma \sin \alpha+\left(\gamma^{\prime}-D\right) \cos \alpha\right) \cos \alpha\right), \\
& \dot{z}=V_{0} \cos \alpha-\frac{1}{R}\left(\gamma \sin \alpha+\left(\gamma^{\prime}-D\right) \cos \alpha\right) \sin \alpha,
\end{aligned}
$$

where $\omega_{0}$ corresponds to the rotation frequency of a spiral wave in two dimensions. At first order in $R^{-1}$ and after the integration over one period of rotation, the dynamics of a scroll ring is (Brazhnik et al. 1987; Alonso et al. 2006b):

$$
\begin{aligned}
& \dot{R}=-\frac{D_{n}}{R}, \\
& \dot{Z}=\frac{D_{b}}{R},
\end{aligned}
$$

where the filament tension $D_{n}$ and the vertical drift parameter $D_{b}$ can be explicitly calculated in terms of the kinematic parameters (Brazhnik et al. 1987; Alonso et al. 2006b; Mikhailov 1995):

$$
\begin{aligned}
& D_{n}=D-\frac{3}{4} \frac{V_{0}}{G_{0}} \frac{\gamma\left(D\left(\gamma^{\prime}-D\right)+\gamma^{2}\right)}{\gamma^{2}+D^{2}}, \\
& D_{b}=\frac{3}{4} \frac{V_{0}}{G_{0}} \frac{\gamma^{2}\left(\gamma^{\prime}-2 D\right)}{\gamma^{2}+D^{2}} .
\end{aligned}
$$


Depending on the value of the parameters the filament tension can change the sign. It follows from Eqs. (11) that filament tension becomes negative under conditions of weak excitability (Alonso et al. 2006b). Scroll waves in the kinematic approach can be formed only if $G_{0}>0$. If $G_{0}<0$ no steadily rotating scroll waves can form and the tip contracts in the course of time. Thus, decreasing the value of $G_{0}$ is equivalent to a reduction of the excitability. We also see that the decrease of $G_{0}$ implies a decrease of $D_{n}$ in Eq. (11) and changes the sign of $D_{n}$ to negative values if $G_{0}$ is sufficiently small.

\subsection{Perturbation Methods}

The main idea of this approach was to assume that a scroll wave can be constructed by placing 2D spirals along a line (filament) slowly varying in space. A periodic rigidly rotating spiral wave was described by $V=V(r, \theta-\omega t)$. The filament dynamics was represented by coordinates along its length $s$ and in normal $p$ and binormal $q$ directions yielding to $V(s, p, q, t)=V_{0}(r, \theta+\phi(s, t)-\omega t)+V_{1}+\cdots$ (where $r$ and $\theta$ are the polar coordinates in the plane $p, q)$.

Using the Frenet-Serret equations (Keener 1988) we can express the threedimensional Laplacian in terms of the coordinates $s, p$ and $q$. We represent the three-dimensional space in the neighborhood of the filament using the coordinate representation $X+p N+q B$. The equations of motion of the filament were derived by rewriting the problem in the coordinate system along the length of the filament $(X)$ and in the normal $(N)$ and binormal $(B)$ directions:

$$
\frac{d X}{d s}=T, \quad \frac{d T}{d s}=k N, \quad \frac{d N}{d s}=-k T+\tau B, \quad \frac{d B}{d s}=-\tau N,
$$

where $T$ is the tangent vector, $k$ the curvature and $\tau$ the torsion of the filament.

Keener derived the equation of filament motion as a function of its curvature and twist (Keener 1988). In general, the equations resulting from the calculation show the evolution of the phase of rotation around the filament $(\phi)$, and the filament $(\mathrm{X})$ evolution in the normal and binormal directions. These equations read:

$$
\begin{aligned}
& \frac{\partial \phi}{\partial t}=\frac{\partial N}{\partial t} \cdot B+\frac{\partial X}{\partial t} \cdot T\left(\frac{\partial \phi}{\partial s}-\tau\right)+b_{1}\left(\frac{\partial^{2} \phi}{\partial s^{2}}-\frac{\partial \tau}{\partial s}\right)+a_{1}\left(\frac{\partial \phi}{\partial s}-\tau\right)^{2}-c_{1} k \\
& \frac{\partial X}{\partial t} \cdot N=b_{2} k-c_{2}\left(\frac{\partial^{2} \phi}{\partial s^{2}}-\frac{\partial \tau}{\partial s}\right)-a_{2}\left(\frac{\partial \phi}{\partial s}-\tau\right)^{2} \\
& \frac{\partial X}{\partial t} \cdot B=c_{3} k-c_{4}\left(\frac{\partial^{2} \phi}{\partial s^{2}}-\frac{\partial \tau}{\partial s}\right)-a_{3}\left(\frac{\partial \phi}{\partial s}-\tau\right)^{2} .
\end{aligned}
$$

For more details, see Keener (1988). Later, in Biktashev et al. (1994), it was shown that due to the symmetry of the scroll ring, the phase $\phi$ is the same everywhere on the ring and the torsion $\tau$ of the filament is zero. Hence, $k=R^{-1}$, and one obtains the following equations for the filament dynamics:

$$
\begin{aligned}
& \dot{R}=-\frac{D_{n}}{R}, \\
& \dot{Z}=\frac{D_{b}}{R},
\end{aligned}
$$


where $\dot{R}$ and $\dot{Z}$ are, respectively, the velocities of the filament in the normal and binormal directions. As in the previous section, the theory predicts a linear dependence of the radius of the scroll ring on the curvature. The parameters $D_{n}=b_{2}$ and $D_{b}=c_{3}$ can be obtained by calculating the adjoint eigenfunctions of the linearization around a stable rotating scroll wave (Keener 1988). Note, however, that the finding of the adjoint solution is not trivial and they can be obtained mainly using numerical methods (Biktasheva et al. 2010).

\subsection{Linear Stability Spectrum of Scroll Waves}

Under some conditions a rigidly rotating scroll wave is stable. In many cases, however, rigidly rotating scroll wave may be unstable. A linear stability analysis allows to detect instabilities of the rigidly rotating scroll waves in a co-rotating reference frame that will be used in the following. First, Eqs. (1) with $D_{w}=0$ are transformed into cylindrical coordinates $(r, \theta, z)$, using $\theta=\theta_{0}-\omega t$ where $\omega$ is the scroll wave rotation frequency:

$$
\begin{aligned}
\left(\frac{\partial}{\partial t}-\omega \frac{\partial}{\partial \theta}\right) V & =D_{V}\left(\nabla_{2 D}^{2}+\frac{\partial^{2}}{\partial z^{2}}\right) V+k I(V, w), \\
\left(\frac{\partial}{\partial t}-\omega \frac{\partial}{\partial \theta}\right) w & =R(V, w) ;
\end{aligned}
$$

where, for simplicity, we have neglected the effects of the twist; for a complete analysis including the twist, see Henry and Hakim (2000). The resulting equation for the steady state is obtained assuming that $V_{0}=V_{0}(r, \theta)$ and $w_{0}=w_{0}(r, \theta)$,

$$
\begin{aligned}
& 0=\left(D_{V} \nabla_{2 D}^{2}+\omega \frac{\partial}{\partial \theta}\right) V_{0}+k I\left(V_{0}, w_{0}\right), \\
& 0=\left(\omega \frac{\partial}{\partial \theta}\right) w_{0}+R\left(V_{0}, w_{0}\right) .
\end{aligned}
$$

These equations are solved numerically to obtain the functions $V_{0}$ and $w_{0}$ (Henry and Hakim 2000) corresponding to the steady state of a rigidly rotating straight scroll in a corotating reference of frame. These functions do not explicitly depend on the z-coordinate.

Next, the stability of the solution obtained from Eqs. (17) can be determined. The linear stability is calculated considering small perturbations around the steady state: $V(r, \theta, z)=V_{0}(r, \theta)+V_{1}(r, \theta) \exp \left(\sigma t-i k_{z} z\right)$ and $w(r, \theta, z)=w_{0}(r, \theta)+$ $w_{1}(r, \theta) \exp \left(\sigma t-i k_{z} z\right)$. The functions $V_{0}, V_{1}, w_{0}$, and $w_{1}$ do not depend explicitly on $\mathrm{z}$ and can be obtained by solving the stability problem of a rigidly rotating spiral wave in a $2 \mathrm{D}$ systems with the same parameters following the method described in Barkley (1992). Introducing these perturbations in Eqs. (16) of the model and considering the steady state obtained from Eqs. (17), the equations obeyed by $V_{1}$ and $w_{1}$ and the growth rate $\sigma$ are obtained:

$$
\begin{aligned}
& \sigma V_{1}=\left(-D_{V} k_{z}^{2}+\omega \frac{\partial}{\partial \theta}+D_{V} \nabla_{2 D}^{2}+\frac{1}{\epsilon} \frac{k \partial I\left(V_{0}, w_{0}\right)}{\partial V}\right) V_{1}+\left(\frac{1}{\epsilon} \frac{k \partial I\left(V_{0}, w_{0}\right)}{\partial w}\right) w_{1}, \\
& \sigma w_{1}=\left(\frac{\partial R\left(V_{0}, w_{0}\right)}{\partial V}\right) V_{1}+\left(\omega \frac{\partial}{\partial \theta}+\frac{\partial R\left(V_{0}, w_{0}\right)}{\partial w}\right) w_{1} .
\end{aligned}
$$


From these equations, the corresponding dispersion relation can be calculated (Henry and Hakim 2002). The dispersion relation is the dependence of the different eigenvalues or growth rates $\sigma$ on $k_{z}$. If the real part of one of the eigenvalues is positive $(\operatorname{Re}(\sigma)>0)$ for a window of values of $k_{z}$, the rigid rotating scroll wave is unstable. One of the eigenvalue pair obtained in the stability analysis of scroll waves corresponding to a perturbation with $k_{z}=0$ is purely imaginary $\pm i \omega$ and is related to the translational invariance of the underlying reaction-diffusion equations. For nonzero values of $k_{z}$, the real part of this eigenvalue may become positive and it is maximal for a particular spatial wavenumber $k_{z}^{\max }$. This instability correspond to the negative filament tension. The dispersion relation permits the numerical calculation of the more unstable mode and it is associated with the critical thickness allowing negative filament tension (Henry and Hakim 2000, 2002).

\section{Control of Negative Filament Tension}

The mechanism responsible for cardiac arrhythmias remains unknown. Negative filament tension may be one of the processes which can be behind the generation of cardiac arrhythmias (Fenton et al. 2002), hence the increasing interest on different control strategies of the instability. Control of nonlinear extended systems has become a well-studied topic during the last years (Mikhailov and Showalter 2006). In the following, we review several methods to control negative filament tension. We account for space-independent global external signals as well as for spatiotemporal forcings.

\subsection{Temporal Forcing}

Application of a weak periodic, spatially uniform external forcing through a temporal modulation of the medium excitability provides an efficient control mechanism for spiral waves (Mikhailov et al. 1994; Mantel and Barkley 1996). Under resonance conditions, i.e., periodic forcing with the same frequency of the spiral rotation, the spiral wave starts to drift along a straight line with the direction determined by the modulation phase. Under nonresonance conditions, the spiral wave performs an externally induced meandering.

We study the effects of a periodic forcing in 3D media by periodically changing the parameter $p_{2}=p_{2}^{o}+p_{f} \cos \left(\omega_{f} t\right)$ of the Barkley model in the numerical simulations. It was shown that rapid forcing with a frequency higher than the rotation frequency of scroll waves converts expanding scroll rings into collapsing ones and suppresses the turbulence in the medium. On the other hand, application of a sufficiently strong forcing with a frequency smaller than the rotation frequency allows to transform collapsing scroll rings into the expanding ones and induce turbulence in the medium (Alonso et al. 2003). 
The kinematic theory, see Sect. 4.1, can be extended to the case of nonresonant forcing for the special case of scroll rings retaining the leading terms of the second order in the forcing amplitude (Alonso et al. 2006b). The result can be expressed as

$$
\begin{aligned}
& \dot{R}=-\frac{D_{n}}{R}-\frac{\xi_{n} p_{f}^{2}}{\Delta \omega R}, \\
& \dot{Z}=\frac{D_{b}}{R}-\frac{\xi_{b} p_{f}^{2}}{\Delta \omega R},
\end{aligned}
$$

where $R$ is the radius of the scroll ring, $Z$ its vertical position, $D_{n}$ and $D_{b}$ are shown in Eqs. (11)-(12), $\Delta \omega$ is the difference between the forcing frequency and the rotation frequency of spiral waves $\left(\Delta \omega=\omega_{f}-\omega\right)$ and the coefficients $\xi_{n}$ and $\xi_{b}$ are positive coefficients determined only by the properties of the excitable medium (Alonso et al. 2006b). The filament tension coefficient of Eq. (11) is effectively renormalized $D_{n}^{e}=$ $D_{n}+\xi_{n} p_{f}^{2} \Delta \omega^{-1}$ by the global nonresonant periodic forcing.

The dynamics of the scroll ring under resonant forcing strongly depends on the phase of the forcing with respect the scroll ring rotation and preclude any type of renormalization of the filament tension. The action of resonant periodic forcing on collapsing scroll rings was numerically studied in Mantel and Barkley (2001). Although the filament tension is not renormalized, global resonant forcing results in an efficient method to induce a strong drift of the filaments which in a closed system produces the termination of the turbulence (Morgan et al. 2008).

\subsection{Spatiotemporal Forcing}

Spatiotemporal forcing of scroll waves results in a more effective method of control. A traveling-wave modulation of the medium's excitability suppresses the turbulent dynamics much faster than a spatial uniform modulation of the medium excitability. Numerical simulations performed with the Barkley model changing the parameter $p_{2}=p_{2}^{o}+p_{f} \cos \left(\vec{k} \vec{r}+\omega_{f} t\right)$ shows the increase of efficiency in removing the turbulent regime (Wu et al. 2006).

A periodic pacing in a localized region of the medium induces the generation of a train of periodic waves. Depending on the frequency of the wave train, the turbulence can be rapidly removed from the system. Slow pacing does not have relevant effects on the dynamics of scroll waves. For fast pacing, however, the frequency of the train is faster than the rotation frequency of the scroll waves. As a result, the unstable filaments are pushed to the boundaries of the system where they disappear (Zhang et al. 2005). There is an optimal frequency which induces the most efficient elimination of the turbulence.

A stochastic forcing of the parameter $p_{2}=p_{2}^{o}+\eta(\vec{x}, t)$ may produce the elimination of the turbulence. The stochastic term $\eta(\vec{x}, t)$ represents a Gaussian white noise with zero mean $\langle\eta(\vec{x}, t)\rangle=0$ and correlations, $\left\langle\eta(\vec{x}, t), \eta\left(\vec{x}^{\prime}, t^{\prime}\right)\right\rangle=2 p_{f}^{2} \delta(t-$ $\left.t^{\prime}\right) \delta\left(\vec{x}-\vec{x}^{\prime}\right)$, where $p_{f}$ is the amplitude of the forcing. A weak stochastic forcing cannot stop the negative filament instability and the filament is unstable; see Figs. 9(a), (b). However, if the noise is above a critical intensity the tension of the filament becomes positive and the filament itself returns to the straight configuration; see Figs. 9(c), (d). The spatiotemporal stochastic forcing enters nonlinearly in the dynamics of the system and renormalizes the parameters of the 
a)

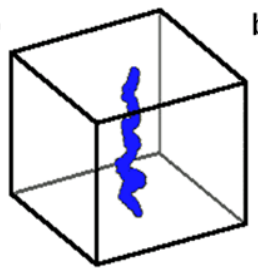

b)

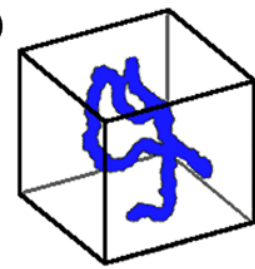

c)

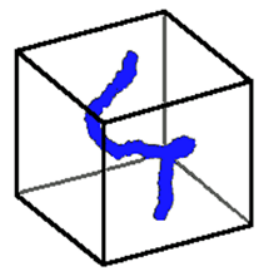

d)

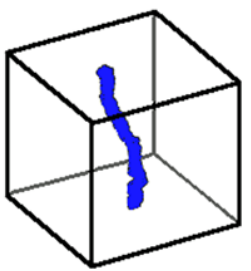

Fig. 9 Dynamics of a scroll wave filament under negative filament tension before $(\mathbf{a}, \mathbf{b})$ and after $(\mathbf{c}, \mathbf{d})$ the introduction of a spatiotemporal stochastic forcing of the parameter $p_{2}$. Figure adapted from Alonso et al. (2004b)

model giving rise to an increase of the effective excitability of the media (Alonso et al. 2004b). As negative filament tension is associated with low excitability, the noise increases the effective excitability and may change the sign of the filament tension. This effect may depend on the specific way the external noise enters into the intrinsic dynamics, for example, spatially static noise may produce the contrary effect, i.e. a decrease of the effective excitability (Alonso et al. 2011; Panfilov 2002).

\section{Negative Filament Tension in Electrophysiologically Realistic Models}

The simple models described so far exhibit negative filament tension. The dynamics under negative filament tension was successfully explained with phenomenological theories of filament dynamics in excitable media. The next step is to study the instability in electro-physiologically realistic models of cardiac tissue.

\subsection{Weak Excitability}

In simple models of excitable media, negative filament tension was found under conditions of weak excitability where the motion of the waves is stable and the interaction between consecutive pulses is weak. The main parameters of the LR1 model (5) that affect excitability are $G_{\mathrm{Na}}$ and $G_{\mathrm{si}}$ corresponding, respectively, to the maximum conductance of sodium and slow inward current. In Alonso and Panfilov (2007), we performed a study of the effects of reducing excitability in the LR1 model on the filament tension. We reduced the value of the maximum conductances $G_{\mathrm{Na}}$ and $G_{\mathrm{si}}$. The effect of the decrease of $G_{\mathrm{si}}$ is a shortening of the action potential duration (APD). If the APD is short, there is less interaction between consecutive pulses. Thus, the trajectories of the spiral tip in 2D and the filament in 3D are rigid or with weak meandering. Therefore, the use of low values of $G_{\mathrm{si}}$, precludes the formation of complex meandering and breakup. The velocity of a traveling wave is almost independent of the value of $G_{\mathrm{si}}$. However, the decrease of $G_{\mathrm{Na}}$ substantially reduces wave velocity, while the APD remains almost constant (Alonso and Panfilov 2007).

We were able to find negative filament tension in the LR1 model. Figure 10 exhibits an example of negative filament tension in the LR1 model. The simulation starts with an initial condition in the form of a scroll wave with a sinusoidally perturbed straight filament; see Fig. 10(a). After several rotations, the filament begins to 
a)

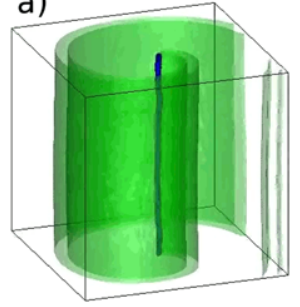

e)

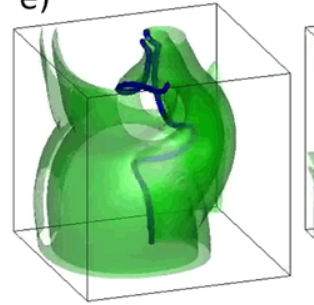

b)

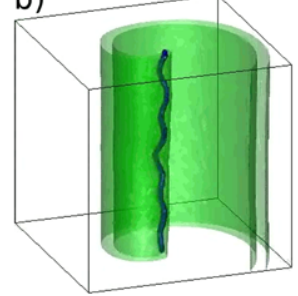

f)

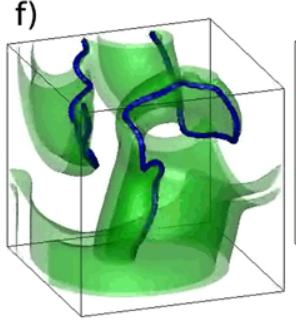

c)

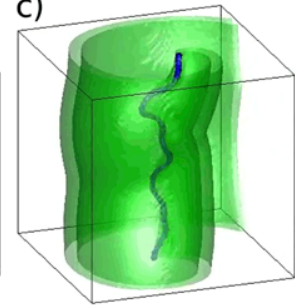

g)

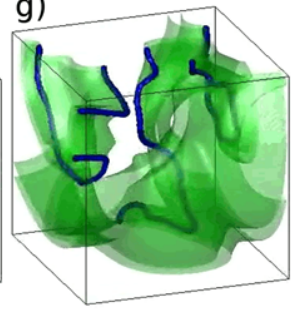

d)

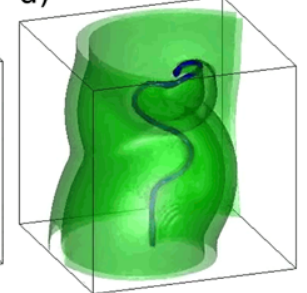

h)

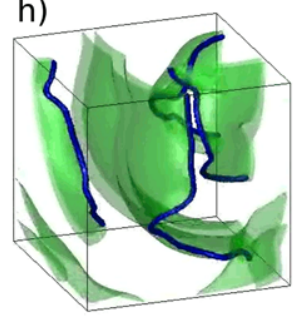

Fig. 10 Evolution of a scroll wave showing negative filament tension in the Luo-Rudy model. Waves and filaments are simultaneously shown. Values of the parameters are $G_{\mathrm{Na}}=3.9 \mathrm{mS} / \mathrm{cm}^{2}$ and $G_{\mathrm{si}}=0.0 \mathrm{mS} / \mathrm{cm}^{2}$

bend; see Fig. 10(b). Length and curvature of the filament increase; see Figs. 10(c) and 10(d). When the filament touches the boundary, see Fig. 10(e), it fragments giving rise to a new scroll wave, see Fig. 10(f). The process of fragmentation persists and gives rise to a complex spatio-temporal pattern of excitation, see Figs. 10(g) and 10(h). This chaotic dynamics persists as long as we perform the simulation. As shown in Fig. 10, new filaments appear and disappear as a result of the interaction with the boundaries and the interaction among the filaments (Alonso and Panfilov 2007). The excitation pattern is equivalent to the dynamics obtained in simple models of excitable media under negative filament tension, shown, for example, in Fig. 4.

In Alonso and Panfilov (2007) we also show that an increase of excitability (e.g. by increase of $G_{\mathrm{Na}}$ ) eliminates negative filament tension. It becomes positive and a filament initially perturbed stabilizes between the opposite boundaries of the system and remains straight for the whole simulation. We also confirmed that in the LR1 model, as in simple models, the negative filament tension disappears if we reduce the thickness of the tissue.

However, the excitability of cardiac tissue in normal conditions is high. Thus, it is essential to find out negative filament tension in cardiac tissue with high excitability.

\subsection{High Excitability}

In Alonso and Panfilov (2008), we performed an extensive search of the phenomenon of negative filament tension for a wide range of parameters of the LR1 model. We were specifically interested in high values of $G_{\mathrm{Na}}$ and $G_{\mathrm{si}}$ corresponding to high excitability of cardiac tissue. For larger values of $G_{\mathrm{Na}}$ and $G_{\mathrm{si}}$, the meandering dynamics of spiral and scroll waves becomes more complex and the dynamics is dominated by several frequencies in contrast to spirals and scrolls with rigid rotation (character- 

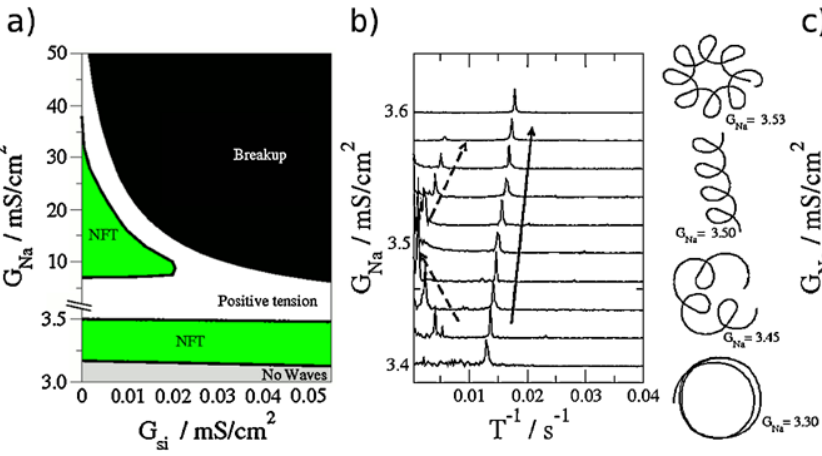

c)

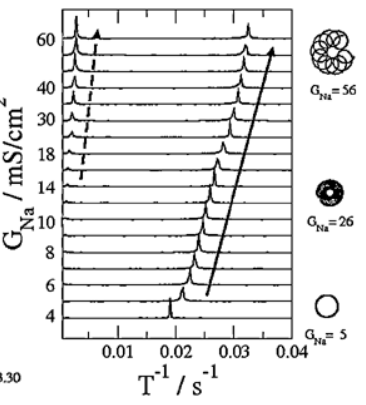

Fig. 11 (a) Phase space of the dynamics of scroll waves in the LR1 model. (b) Fourier spectrum of the spiral tip motion for 2D spiral waves in low-excitable media for different values of $G_{\mathrm{Na}}$. Panels show particular examples of spiral dynamics at low excitability. (c) Fourier spectrum of the spiral tip motion for 2D spiral waves in high-excitable media for different values of $G_{\mathrm{Na}}$. Panels show particular examples of spiral dynamics at high excitability. The simulations here are performed with LR1 model, where $j$ is clamped to 1

ized by a single frequency) and simple meandering (characterized by two frequencies). To reduce the number of frequencies and keeping the main characteristics of wave propagation, we fixed the gating variable $\mathrm{j}$ to a value 1 (Qu et al. 2000b), representing a slow inactivation of $I_{\mathrm{Na}}$ that permits the elimination of several frequencies in the meandering motion, resulting in simple meandering of spiral waves.

In Fig. 11(a) the behaviors the LR1 model is shown in the parameter spanned by the conductivities $G_{\mathrm{Na}}$ and $G_{\mathrm{si}}$, where the variable $\mathrm{j}$ is clamped to 1 . At high values of $G_{\text {si }}$ spiral breakup becomes rapidly dominant. The usual region of negative filament tension is obtained at the low excitability limit, for the range $3.1<G_{\mathrm{Na}}<$ $3.5 \mathrm{mS} / \mathrm{cm}^{2}$ for a wide range of values of $G_{\text {si }}$. This region corresponds to the classical regime of negative filament tension in media with low excitability which we have previously discussed in this review.

We have also found a region of the parameter space where scroll rings can expand even if the medium is highly excitable. This second region of negative filament tension is found at $8<G_{\mathrm{Na}}<35 \mathrm{mS} / \mathrm{cm}^{2}$ for $G_{\mathrm{si}}=0$, and its size decreases with the increase of parameter $G_{\mathrm{Si}}$, see phase diagram in Fig. 11(a). Numerical simulations of scroll rings in this region show that the rate of expansion is low, the growth, however, is robust and is not due to numerical or boundary effects (Alonso and Panfilov 2008). In our numerical simulations this weak negative filament tension of scroll rings was not able to produce a turbulent dynamics.

The expansion of scroll rings at low excitability is related with the transition between inward and outward meandering. Figure 11(b) shows the Fourier analysis of the spiral tip motion for different values of $G_{\mathrm{Na}}$ and several examples of meandering patterns of the spiral tip. For each value of $G_{\mathrm{Na}}$ typically two frequencies are shown: the large frequency corresponds to the spiral rotation $\omega$ and the small frequency to the meandering $\Delta \omega_{m}=\omega_{m}-\omega$. For low values of $G_{\mathrm{Na}}$ spirals perform an inward meandering which evolves into a resonant drift $\left(\omega_{m}=\omega\right)$, and into an outward meandering by increasing the parameter $G_{\mathrm{Na}}$. The region with inward (outward) meandering corresponds to negative (positive) tension. 
Expansion of scroll rings at high excitability is partially related to a region of inward meandering of 2D spiral waves. Figure 11(c) shows the appearance of a new frequency in the Fourier spectrum by the increase of the parameter $G_{\mathrm{Na}}$. For $G_{\mathrm{Na}}=$ $5 \mathrm{mS} / \mathrm{cm}^{2}$ spiral waves rotate rigidly. The increase of $G_{\mathrm{Na}}$ produces a secondary frequency inducing an inward meandering. Note that the dependence of this new frequency on the parameter $G_{\mathrm{Na}}$ in Fig. 11(c) differs from the typical meandering instability shown in Fig. 11(b).

These results highlight the relation between inward meandering in two-dimensional media and the tension of three-dimensional filaments. In contrast to the low excitability case, the boundaries of negative filament tension do not precisely coincide with the boundaries of the different meandering regimes.

\section{Discussion}

In this review, we have considered studies of negative filament tension performed numerically in simple models of excitable media and in detailed ionic models of cardiac tissue as well as corresponding analytical approaches. Two main conclusions are that negative filament tension typically results in the onset of turbulence in 3D excitable media and that it normally occurs in media with low excitability. We also have reported on negative filament tension in media with high excitability. The characterization of the instability under such condition requires, however, additional studies. The negative filament tension instability does not develop if the thickness of the medium is below a critical value, which is determined by the maximal spatial wavenumber of an unstable mode. Numerical results also show a relation between the characteristics of spiral wave meandering in 2D and the negative filament tension of straight scrolls or scroll rings in 3D. Negative line tension in 3D is usually found at parameter values where spirals show inward meandering in 2D. Analytical approaches can explain the negative filament tension. It is, however, difficult to predict its onset for a particular model of cardiac tissue. Such approaches require either the knowledge of some phenomenological quantities which cannot be explicitly obtained from the parameters of the model or they are based on the knowledge of the adjoint eigenfunctions of the linearization around a stable rotating scroll wave which can be only done using numerical methods. Furthermore, it would be important to extend the analytical description from rigidly rotating to meandering spirals and scrolls.

Much remains to be done to explore the influence of geometry on the development of negative line tension for realistic heart shapes. In an influential paper, Winfree pointed out that ventricular fibrillation was absent in small animals with small hearts (Winfree 1994) and that ventricular fibrillation can occur if the thickness of the ventricular wall of the heart exceeds some critical value. From this, he concluded that genuine 3D effects like negative filament tension may be crucial in the development of fibrillation. Nowadays, fibrillation can be induced in small animal hearts (e.g., mouse or rat heart), but in many cases this requires application of drugs which reduces the duration of the wave. Thus, thickness of the heart should be considered relative to the characteristic wavelength of the scroll wave (Panfilov 2006). In addition, it is not known in which cases VF does indeed occur via the negative filament tension mechanism. 
Although there is not yet a direct evidence of negative filament tension in the heart, several indirect observations point towards a role of the negative filament tension mechanism. The development of the negative filament tension instability in the heart (see Fig. 8) produces surface activity patterns similar to those observed in the heart during the development of ventricular fibrillation (Zaitsev 2008). Furthermore, it is widely recognized that many cardiac arrhythmias occur in conditions of reduced excitability of the heart, e.g., during ischemia. The reduction of excitability favors the appearance of negative filament tension. However, our studies of cardiac models (Alonso and Panfilov 2007) showed that it occurs only in cases of extreme excitability decrease (nonphysiologically weak sodium and calcium conductances, corresponding to low values of the parameters $G_{\mathrm{Na}}$ and $G_{\mathrm{si}}$ ) and it is not clear if such conditions are realizable in the heart. In that respect, an interesting observation of negative filament tension at high excitability was reported in Alonso and Panfilov (2008). However, the effect needs to be verified in other models of cardiac tissue.

Another important topic on wave propagation in cardiac tissue is the effect of anisotropy. Recent analytical studies (Verschelde et al. 2007; Dierckx et al. 2009) show that nonhomogeneous anisotropy can substantially affect scrolls and may generate the complex dynamics of scroll waves observed in numerical simulations (Fenton and Karma 1998b). Most of the studies on effects of anisotropy were performed for positive filament tension (Panfilov and Keener 1995b; Qu et al. 2000a). Numerical simulations for parameters value close to the boundary where negative filament tension sets show that anisotropy induces negative filament tension (Mi and Ping 2009).

Another possible factor which may contribute to an emergence of negative filament tension in the heart is cardiac contraction. The mechanical deformation of the heart during the contraction interacts with the propagation of the electrical waves which is known as a mechano-electrical feedback. Recent studies showed that the mechano-electrical feedback is normally associated with a phenomenon of accommodation which decreases the excitability of the tissue (Panfilov et al. 2007). Thus, we expect that it should make negative filament tension more pronounced. However, as was shown in Panfilov et al. (2007), the mechano-electrical feedback also induces a resonant drift of the spiral waves which may substantially affect filament tension in a way that is difficult to predict from the results of two-dimensional simulations, and hence would require a computationally expensive study based on three-dimensional simulations.

Negative filament tension is one of the possible mechanisms to generate turbulence in excitable media. Another widely discussed mechanism are instabilities that cause spiral wave breakup in two dimensions (Fenton et al. 2002; Bär and Brusch 2004), for example, the previously mentioned breakup by steep restitution curves (Panfilov and Holden 1990; Karma 1993). The main difference between them is that negative tension in simple models is associated with low excitability, where the rotation period of the scroll wave is substantially longer than the refractory time of the medium. On the contrary, spiral breakup is typically associated with highly excitable media, where the rotation period of spiral and scroll waves is close to the refractory period and the velocity of the waves is substantially above the values found at propagation failure. Recently, both types of turbulences were studied in order to differentiate these two mechanisms from each other. It was shown that the statistical properties of the 
turbulent defect dynamics by negative filament tension are different from those generated by instabilities associated with 2D mechanism of spiral breakup (Davidsen et al. 2008; Reid et al. 2011). A similar approach may allow researchers to distinguish between these two mechanisms in experiments because three-dimensional visualization of the turbulence on the heart is still not available and only the chaotic surface electrical activity is the accessible. Three-dimensional visualization is, however, possible in chemical excitable systems using tomographic techniques. It has permitted the visualization of negative filament tension in the Belousov-Zhabotinsky (BZ) reaction for scroll waves (Luengviriya et al. 2008) and rings (Bánsági and Steinbock 2007) as already predicted by numerical simulations of the Oregonator model of the BZ reaction (Alonso et al. 2006a).

In summary, negative filament tension of scroll wave induces the formation of a turbulent dynamics in simple and electrophysiologically realistic models of cardiac tissue. The turbulent pattern obtained during this state is reminiscent of the chaotic and irregular wave dynamics observed in experiments with cardiac tissue or hearts during VF. The emergence of negative filament tension in real cardiac tissue may be closely related with the appearance of ischemia in cardiac tissue.

Acknowledgements Financial support by the Deutsche Forschungs-gemeinschaft (DFG) within the framework of SFB 910 (Control of Self-Organizing Nonlinear Systems) is acknowledged. We are grateful to A.S. Mikhailov, F. Sagués, H. Engel, and M.J.B. Hauser for valuable discussions about scroll wave dynamics.

\section{References}

Aliev, R. R., \& Panfilov, A. V. (1996). A simple two-variable model of cardiac excitation. Chaos Solitons Fractals, 7, 293-301.

Alonso, S., \& Panfilov, A. V. (2007). Negative filament tension in the Luo-Rudy model of cardiac tissue. Chaos, 17, 015102 .

Alonso, S., \& Panfilov, A. V. (2008). Negative filament tension at high excitability in a model of cardiac tissue. Phys. Rev. Lett., 100, 218101.

Alonso, S., Sagués, F., \& Mikhailov, A. S. (2003). Taming Winfree turbulence of scroll waves in excitable media. Science, 299, 1722-1725.

Alonso, S., Kähler, R., Sagués, F., \& Mikhailov, A. S. (2004a). Expanding scroll rings and negative tension turbulence in a model of excitable media. Phys. Rev. E, 70, 056201.

Alonso, S., Sancho, J. M., \& Sagués, F. (2004b). Suppression of scroll wave turbulence by noise. Phys. Rev. E, 70, 067201(R).

Alonso, S., Sagués, F., \& Mikhailov, A. S. (2006a). Negative-tension instability of scroll waves and Winfree turbulence in the Oregonator model. J. Phys. Chem. A, 110, 12063-12071.

Alonso, S., Sagués, F., \& Mikhailov, A. S. (2006b). Periodic forcing of scroll rings and control of Winfree turbulence. Chaos, 16, 023124.

Alonso, S., Kapral, R., \& Bär, M. (2009). Effective medium theory for reaction rates and diffusion coefficients of heterogeneous systems. Phys. Rev. Lett., 102, 238302.

Alonso, S., Bär, M., \& Panfilov, A. V. (2011). Effects of reduced discrete coupling on filament tension in excitable media. Chaos, 21, 013118.

Bánsági, T., \& Steinbock, O. (2007). Negative filament tension of scroll rings in an excitable system. Phys. Rev. $E, 76,045202(\mathrm{R})$.

Bär, M., \& Brusch, L. (2004). Breakup of spiral waves caused by radial dynamics: Eckhaus and finite wavenumber instabilities. New J. Phys., 6, 5.

Bär, M., \& Eiswirth, M. (1993). Turbulence due to spiral breakup in a continuous excitable medium. Phys. Rev. E, 48, R1635-R1637. 
Barkley, D. (1992). Linear stability analysis of rotating spiral waves in excitable media. Phys. Rev. Lett., 68, 2090-2093.

Barkley, D., Kness, M., \& Tuckerman, L. S. (1990). Spiral-wave dynamics in a simple model of excitable media: the transition from simple to compound rotation. Phys. Rev. A, 42, 2489-2492.

Biktashev, V. N. (1998). A three-dimensional autowave turbulence. Int. J. Bifurc. Chaos Appl. Sci. Eng., $8,677$.

Biktashev, V. N., Holden, A. V., \& Zhang, H. (1994). Tension of organizing filaments of scroll waves. Philos. Trans. R. Soc. Lond. Ser. A, 347, 611.

Biktasheva, I. V., Barkley, D., Biktashev, V. N., \& Foulkes, A. J. (2010). Computation of the drift velocity of spiral waves using response functions. Phys. Rev. E, 81, 066202.

Brazhnik, P. K., Davydov, V. A., Zykov, V. S., \& Mikhailov, A. S. (1987). Vortex rings in excitable media. Sov. Phys. JETP, 66, 984.

Clayton, R. H., Bernus, O., Cherry, E. M., Dierckx, H., Fenton, F. H., Mirabella, L., Panfilov, A. V., Sachse, F. B., Seemann, G., \& Zhang, H. (2011). Models of cardiac tissue electrophysiology: progress, challenges and open questions. Prog. Biophys. Mol. Biol., 104, 22-48.

Davidsen, J., Zhan, M., \& Kapral, R. (2008). Filament-induced surface spiral turbulence in threedimensional excitable media. Phys. Rev. Lett., 101, 208302.

Dierckx, H., Bernus, O., \& Verschelde, H. (2009). A geometric theory for scroll wave filaments in anisotropic excitable media. Physica D, 238, 941-950.

Dowle, M., Mantel, R. M., \& Barkley, D. (1997). Fast simulations of waves in three-dimensional excitable media. Int. J. Bifurc. Chaos Appl. Sci. Eng., 7, 2529-2546.

Fenton, F. H., \& Karma, A. (1998a). Vortex dynamics in three-dimensional continuous myocardium with fiber rotation: filament instability and fibrillation. Chaos, 8, 20.

Fenton, F., \& Karma, A. (1998b). Fiber-rotation-induced vortex turbulence in thick myocardium. Phys. Rev. Lett., 81, 481-484.

Fenton, F. H., Cherry, E. M., Hastings, H. M., \& Evans, S. J. (2002). Multiple mechanisms of spiral wave breakup in a model of cardiac electrical activity. Chaos, 12, 852.

Gray, R. A., \& Jalife, J. (1998). Ventricular fibrillation and atrial fibrillation are two different beasts. Chaos, 8,65 .

Gray, R. A., Jalife, J., Panfilov, A. V., Baxter, W. T., Cabo, C., Davidenko, J. M., \& Pertsov, A. M. (1995). Nonstationary vortexlike reentrant activity as a mechanism of polymorphic ventricular tachycardia in the isolated rabbit heart. Circ. Res., 91, 2454-2469.

Henry, H. (2004). Spiral wave drift in an electric field and scroll wave instabilities. Phys. Rev. E, 70, 026204.

Henry, H., \& Hakim, V. (2000). Linear stability of scroll waves. Phys. Rev. Lett., 85, 5328.

Henry, H., \& Hakim, V. (2002). Scroll waves in isotropic excitable media: linear instabilities, bifurcations, and restabilized states. Phys. Rev. E, 65, 046235.

Hodgkin, A. L., \& Huxley, A. F. (1952). A quantitative description of membrane current and its application to conduction and excitation in nerve. J. Physiol., 117, 500-544.

Izhikevich, E. M., \& FitzHugh, R. (2006). FitzHugh-Nagumo model. Scholarpedia, 1, 1349.

Karma, A. (1990). Meandering transition in two-dimensional excitable media. Phys. Rev. Lett., 65, 2824.

Karma, A. (1993). Spiral breakup in model equations of action potential propagation in cardiac tissue. Phys. Rev. Lett., 71, 1103-1106.

Keener, J. P. (1988). The dynamics of three-dimensional scroll waves in excitable media. Physica D, 31, 269.

Keener, J. P., \& Sneyd, J. (1998). Mathematical physiology. New York: Springer.

Kléber, A., \& Rudy, Y. (2004). Basic mechanisms of cardiac impulse propagation and associated arrhythmias. Physiol. Rev., 84, 431-488.

Luengviriya, C., Storb, U., Lindner, G., Müller, S. C., Bär, M., \& Hauser, M. J. B. (2008). Scroll wave instabilities in an excitable chemical medium. Phys. Rev. Lett., 100, 148302.

Luo, C. H., \& Rudy, Y. (1991). A model of the ventricular cardiac action potential. Depolarization, repolarization, and their interaction. Circ. Res., 68, 1501.

Mantel, R. M., \& Barkley, D. (1996). Periodic forcing of spiral waves in excitable media. Phys. Rev. E, 54,4791

Mantel, R. M., \& Barkley, D. (2001). Parametric forcing of scroll-wave patterns in three-dimensional excitable media. Physica D, 149, 107.

Meron, E. (1992). Pattern formation in excitable media. Phys. Rep., 218, 1-66.

Mi, J., \& Ping, M. (2009). Vortex turbulence due to the interplay of filament tension and rotational anisotropy. Chin. Phys. Lett., 26, 074703. 
Mikhailov, A. S. (1995). Three-dimensional kinematic. Chaos Solitons Fractals, 5, 673.

Mikhailov, A. S., \& Showalter, K. (2006). Control of waves, patterns and turbulence in chemical systems. Phys. Rep., 425, 79-194.

Mikhailov, A. S., Davydov, V. A., \& Zykov, V. S. (1994). Complex dynamics of spiral waves and motion of curves. Physica D, 70, 1 .

Moe, G. K., Rheinbolt, W. C., \& Abildskov, J. A. (1964). A computer model of atrial fibrillation. Am. Heart J., 67, 200-220.

Morgan, S. W., Biktasheva, I. V., \& Biktashev, V. N. (2008). Control of scroll-wave turbulence using resonant perturbations. Phys. Rev. E, 78, 046207.

Nielsen, P. M. F., LeGrice, I. J., Smail, B. H., \& Hunter, P. J. (1991). A mathematical model of the geometry and fibrous structure of the heart. Am. J. Physiol., 260, H1365-H1378.

Noble, D., \& Rudy, Y. (2001). Models of cardiac ventricular action potentials: iterative interaction between experiment and simulation. Philos. Trans. R. Soc. Lond. A, 359, 1127-1142.

Panfilov, A. V. (1999). Three-dimensional organization of electrical turbulence in the heart. Phys. Rev. E, 59, R6251-R6254.

Panfilov, A. V. (2002). Spiral breakup in an array of coupled cells: the role of the intercellular conductance. Phys. Rev. Lett., 88, 118101.

Panfilov, A. V. (2006). Is heart size a factor in ventricular fibrillation? Or how close are rabbit and human hearts? Heart Rhythm, 3, 862.

Panfilov, A. V., \& Hogeweg, P. (1993). Spiral breakup in a modified FitzHugh-Nagumo model. Phys. Lett. A, 176, 295-299.

Panfilov, A. V., \& Holden, A. V. (1990). Self-generation of turbulent vortices in a two-dimensional model of cardiac tissue. Phys. Lett. A, 151, 23-26.

Panfilov, A. V., \& Holden, A. V. (1993). Computer simulation of re-entry sources in myocardium in two and three dimensions. J. Theor. Biol., 161, 271-285.

Panfilov, A. V., \& Keener, J. P. (1995a). Re-entry in an anatomical model of the heart. Chaos Solitons Fractals, 5, 681-689.

Panfilov, A. V., \& Keener, J. P. (1995b). Re-entry in three-dimensional Fitzhugh-Nagumo medium with rotational anisotropy. Physica D, 84, 545-552.

Panfilov, A. V., \& Pertsov, A. M. (1984). Vortex rings in 3-dimensional active medium described by reaction diffusion equations. Dokl. Akad. Nauk SSSR, 274, 1500-1503.

Panfilov, A. V., \& Pertsov, A. (2001). Ventricular fibrillation: evolution of the multi-wavelet hypothesis. Philos. Trans. R. Soc. Lond. A, 359, 1315-1325.

Panfilov, A. V., \& Rudenko, A. N. (1987). Two regimes of the scroll ring drift in the three-dimensional active media. Physica D, 28, 215.

Panfilov, A. V., Keldermann, R. H., \& Nash, M. P. (2007). Drift and breakup of spiral waves in reactiondiffusion-mechanics systems. Proc. Natl. Acad. Sci. USA, 104, 7922-7926.

Pertsov, A. M., \& Jalife, J. (2002). Cardiac electrophysiology. From cell to bedside. Philadelphia: Saunders.

Qu, Z., Kil, J., Xie, F., Garfinkel, A., \& Weisse, J. N. (2000a). Scroll wave dynamics in a three-dimensional cardiac tissue model: roles of restitution, thickness, and fiber rotation. Biophys. J., 78, 2761-2775.

Qu, Z., Xie, F., Garfinkel, A., \& Weiss, J. N. (2000b). Origins of spiral wave meander and breakup in a two-dimensional cardiac tissue model. Ann. Biomed. Eng., 28, 755-771.

Reid, J. C., Chaté, H., \& Davidsen, J. (2011). Filament turbulence in oscillatory media. Europhys. Lett., 94, 68003.

Shaw, R. M., \& Rudy, Y. (1997). Roles of the sodium and L-type calcium currents during reduced excitability and decreased gap junction coupling. Circ. Res., 35, 256.

Siegert, F., \& Weijer, C. J. (1992). Three-dimensional scroll waves organize dictyostelium slugs. Proc. Natl. Acad. Sci. USA, 89, 6433.

ten Tusscher, K. H. W. J., Noble, D., Noble, P. J., \& Panfilov, A. V. (2004). A model for human ventricular tissue. Am. J. Physiol., Heart Circ. Physiol., 286, H1573-H1589.

ten Tusscher, K. H. W. J., Bernus, O., Hren, R., \& Panfilov, A. V. (2006). Comparison of electrophysiological models for human ventricular cells and tissues. Prog. Biophys. Mol. Biol., 90, 326-345.

Verschelde, H., Dierckx, H., \& Bernus, O. (2007). Covariant stringlike dynamics of scroll wave filaments in anisotropic cardiac tissue. Phys. Rev. Lett., 99, 168104.

Weiss, J. N., Chen, P.-S., Qu, Z., Karagueuzian, H. S., \& Garfinkel, A. (2000). Ventricular fibrillation: how do we stop the waves from breaking? Circ. Res., 87, 1103-1107. 
Wiener, N., \& Rosenblueth, A. (1946). The mathematical formulation of the problem of conduction of impulses in a network of connected excitable elements, specifically in cardiac muscle. Arch. Inst. Cardiol. Méx., 16, 205-265.

Winfree, A. T. (1972). Spiral waves of chemical activity. Science, 175, 634-636.

Winfree, A. T. (1973). Scroll-shaped waves of chemical activity in three dimensions. Science, 181, 937939.

Winfree, A. T. (1994). Electrical turbulence in three-dimensional heart muscle. Science, 266, 1003.

Wu, N. J., Zhang, H., Ying, H. P., Cao, Z., \& Hu, G. (2006). Suppression of Winfree turbulence under weak spatiotemporal perturbation. Phys. Rev. E, 73, 060901(R).

Zaikin, A. N., \& Zhabotinsky, A. M. (1970). Concentration wave propagation in two-dimensional liquidphase self-oscillating system. Nature, 225, 535-537.

Zaitsev, A. (2008). Personal communication.

Zaritski, R. M., \& Pertsov, A. M. (2005). Stable spiral structures and their interaction in two-dimensional excitable media. Phys. Rev. E, 66, 066120.

Zaritski, R. M., Mironov, S. F., \& Pertsov, A. M. (2004). Intermittent self-organization of scroll wave turbulence in three-dimensional excitable media. Phys. Rev. Lett., 92, 168302.

Zhang, H., Cao, Z., Wu, N. J., Ying, H. P., \& Hu, G. (2005). Suppress Winfree turbulence by local forcing excitable systems. Phys. Rev. E, 94, 188301. 